\title{
EP-130
}

\section{Hepatolithiasis long term complication of choledochal cyst resection: Rase report}

\author{
Ana Isabel ARGUETA*, Francisco DE LEON, José CONTRERAS, Jimmy IXCAYAU, Haroldo PAIZ, Estuardo PORRAS, Erick SOTO \\ Department of Surgery, Guatemala
}

Introduction: Choledochal cysts (CCs) are uncommon with an incidence that ranges from 1 in 100,000-150,000 in Western populations, to 1-1,000 in some Asian populations. The cyst can become complicated with stone disease, pancreatitis, cholangitis and malignancy of the biliary tree; therefore it has to be treated by operative resection of the bile duct, cholecystectomy, and biliodigestive reconstruction, irrespective of the age at presentation. In the long term follow up, postoperative intrahepatic stone and cholangitis present serious complications. Hepatolithiasis is likely to develop secondary to a postoperative anastomotic stricture.

Methods: We present a case report of a 23-year-old male with recurrent episodes of cholangitis and a past surgical history of complete choledocal cyst excision with bilioenteric anastomosis 16 years ago with no long term follow up. The diagnosis of anastomotic stenosis and hepatolithiasis was confirmed by magnetic resonance cholangiopancreatography. Percutaneous treatment was not possible. Patient was taken to surgery where 28 bile stones were removed from choledoco, common hepatic and left hepatic duct. Intraoperative choledochoscopy confirmed stone clearance from the intrahepatic and extrahepatic ductal system. Biopsy was taken and a hepaticojejunostomy according to Hepp-Couinaud performed.

Results: Biopsy reported negative for malignancy. Patient was discharged with no postoperative complications.

Conclusions: Close follow-up after CCs surgical treatment is important, moreover, these patients have a need for long term follow up to detect hepatolithiasis as soon as possible. Combined surgical procedure (stone extraction, on-table choledochoscopy and bilio-enteric drainage) is an acceptable option for hepatolithiasis. 